\title{
Potency of Carbapenems for the Prevention of Carbapenem- Resistant Mutants of Pseudomonas aeruginosa
}

\author{
The High Potency of a New Carbapenem Doripenem
}

\author{
Shihomi Sakyo, Haruyoshi Tomita, Koichi Tanimoto, Shuhei Fujimoto, Yasuyoshi Ike
}

Received: February 2, 2006 / Accepted: March 29, 2006

(C) Japan Antibiotics Research Association

\begin{abstract}
The potencies of the carbapenems; doripenem (DRPM), meropenem (MEPM) and imipenem (IPM) in preventing the emergence of carbapenem-resistant mutants were examined in Pseudomonas aeruginosa strains. The carbapenems predominantly selected carbapenem-resistant mutants or carbapenem mutants with reduced susceptibilities that were specifically resistant to carbapenems and had arisen as a result of the reduced level of expression of the outer membrane protein with a molecular weight of about 48,000 (OprD). The potency of carbapenems in preventing the growth of the mutants differed for DRPM, MEPM and IPM. The isolation frequency of the mutant was examined on agar plates containing each of the carbapenems at a concentration of $1 / 2$ or $1 / 4$ MIC of each carbapenem for that mutant. Mutants were not selected on agar containing DRPM at a frequency of greater than $10^{-9}$ per cell per generation, whereas mutants of each strain were selected on agar containing MEPM or IPM at frequencies of $10^{-7}$ to $10^{-9}$ per cell per generation. The drug concentrations and the drug concentration range for the selective increase of carbapenem resistant mutants in the broth culture containing each carbapenem differed for each carbapenem. DRPM exhibited both the lowest drug concentration and the narrowest range of drug concentration for selection of the carbapenem-resistant mutants. The results shown in this report indicated that DRPM exhibited the greatest ability to prevent the emergence of the mutant.
\end{abstract}

Keywords carbapenem, doripenem, carbapenem-resistant mutant, outer membrane protein, prevention of mutation

\section{Introduction}

Pseudomonas aeruginosa is an opportunistic pathogen for immunocompromised patients. The organism has both an intrinsic and acquired resistance to many antibiotics [1,2], and treatment of infection by this organism is difficult. Carbapenems have a high potency against a broad spectrum of organisms and are one of the most active groups of $\beta$ lactam antibiotics against $P$. aeruginosa.

There is a group of carbapenem resistant $P$. aeruginosa strains that is specifically resistant to carbapenems and which does not show cross resistance to other $\beta$-lactams or other antibiotics. This type of imipenem (IPM)-resistant $P$. aeruginosa mutant was first isolated during clinical trials of IPM for the treatment of serious infections caused by $P$. aeruginosa [3]. The IPM-resistant mutants of $P$. aeruginosa were found to lack a $45 \mathrm{KD}$ to $49 \mathrm{KD}$ protein [3 6] in the outer membrane protein D2 (OprD), which forms a channel specific to IPM and its structural analogues [7].

In this study, we investigated the potency of doripenem (DRPM) [8 12], meropenem (MEPM) and IPM in preventing the emergence of carbapenem-resistant mutants under laboratory conditions.
Y. Ike (Corresponding author), S. Sakyo, H. Tomita, S. Fujimoto: Department of Bacteriology and Bacterial Infection Control, Gunma University Graduate School of Medicine, Showamachi 3-39-22, Maebashi, Gunma 371-8511, Japan,

E-mail: yasuike@med.gunma-u.ac.jp
K. Tanimoto, Y. Ike: Laboratory of Bacterial drug Resistance, Gunma University Graduate School of Medicine, Showa-machi 339-22, Maebashi, Gunma 371-8511, Japan 


\section{Materials and Methods}

\section{Bacteria, and Media}

One hundred and forty-four clinical P. aeruginosa isolates were examined for carbapenem resistance. Of the 144 strains, 100 strains were isolated from Gunma University Hospital, 30 strains from Isesaki City Hospital, Isesaki City, Japan, and 14 strains from Gunma Chuo-hospital, Maebashi, Japan. Mueller-Hinton (MH) (Nissui, Tokyo, Japan) broth and MH agar or antibiotic medium 3 (AB3) (Difco, Detroit, Mich.) and AB3 agar were used to test the MICs for each antibiotic. Agar plates were prepared by adding $1.5 \%$ agar to broth medium. All bacterial strains were grown at $37^{\circ} \mathrm{C}$. MICs obtained using $\mathrm{MH}$ medium and AB3 medium were not essentially different (data not shown). Mutation frequencies and the kinetics of bacterial growth were examined for each medium. As a result, AB3 medium was used throughout this study for its capacity to maintain a stable $\mathrm{pH}$ during course of bacterial growth.

\section{Determination of MICs}

MIC was determined by the agar dilution method. Overnight cultures of the strains grown in broth were diluted 100 times with fresh broth. One loopful of each dilution was plated on agar plates containing two-fold serially-diluted antibiotics. The plates were incubated for 18 hours at $37^{\circ} \mathrm{C}$.

\section{Antibiotics}

Antibiotics used in this study were as follows: DRPM (a new carbapenem that had been developed by Shionogi Pharmaceuticals, Osaka) [8 12], MEPM (Sumitomo Pharmaceuticals, Osaka), IPM (Banyu Pharmaceuticals, Tokyo), piperacillin (PIPC, Toyama Chemicals, Tokyo), ceftazidime (CAZ, Tanabe Pharmaceuticals, Osaka) and gentamicin (GM, Schering-Plough K.K., Osaka) and ofloxacin (OFLX, Daiichi Seiyaku Co., Ltd., Tokyo).

\section{Isolation Frequency of Mutant}

The frequency of isolation of the mutant was estimated using the following equation for mutation frequency [13]: Mutation frequency per cell per generation $=[\Pi-\Pi 0 / 1 \mathrm{n}(P /$ $P 0)]$ where $\Pi 0$ and $P 0$ are, respectively, the proportion of mutant cells and the total number of cells in the culture at the start of the experiment, and $\Pi$ is the proportion of mutant cells after incubation when the number of total cells has risen to $P$.

\section{Analysis of Outer Membrane Proteins (OMPs)}

The OMPs of all strains were analyzed by SDS-PAGE of the sarkosyl insoluble fraction of membrane-preparation as described by Spratt [14]. Throughout this study, the OMPs of the carbapenem-resistant mutants of the representative strains obtained in each experiment were examined to confirm whether the mutants lacked, or had reduced amount of, OMP of molecular weight 45,000 49,000 (data not shown).

\section{Results}

\section{Distribution of Carbapenem MICs among P. aeruginosa Isolates}

The carbapenem resistance levels (MICs) of the 144 strains of $P$. aeruginosa were examined. There was a bi-modal distribution of the MICs for each drug in these strains (data not shown). However, the MICs showed a continuous distribution, and there was no complete break between the bi-modal distributions. The MICs of DRPM for these strains ranged from 0.1 to $1.6 \mu \mathrm{g} / \mathrm{ml}$, and from 1.6 to $12.5 \mu \mathrm{g} / \mathrm{ml}$. The MICs to MEPM ranged from 0.1 to $1.6 \mu \mathrm{g} / \mathrm{ml}$, and from 1.6 to $50 \mu \mathrm{g} / \mathrm{ml}$. The MICs of IPM for these strains ranged from 0.1 to 6.3 , and from 6.3 to $100 \mu \mathrm{g} / \mathrm{ml}$. The $\mathrm{MIC}_{90}$ values of DRPM, MEPM, and IPM were $3.2,6.3$, and $25 \mu \mathrm{g} / \mathrm{ml}$, respectively. Ten carbapenem susceptible strains were chosen for further study. The susceptibilities to carbapenems and other antimicrobial agents by the ten strains are shown in Table 1 .

\section{Appearance of Colonies of Carbapenem-resistant Mutants within the Zone of Growth Inhibition}

Figure 1 shows a typical result of the growth inhibition of the $P$. aeruginosa GP17 strain. The MICs of DRPM, MEPM and IPM for the parent strain GP17 were $0.1,0.1$ and $1.6 \mu \mathrm{g} / \mathrm{ml}$, respectively.

In experiments with IPM and MEPM, there were several colonies within the clear zone of growth inhibition, or on the border between the clear zone of growth inhibition and the zone of cell growth. However, there were no colonies within the clear zone of growth inhibition when DRPM was used. The colonies that grew within the clear zone of growth inhibition in the MEPM or IPM experiments, or on the border in the MEPM experiment showed MIC values of $1.6 \mu \mathrm{g} / \mathrm{ml}$ to DRPM, $3.2 \mu \mathrm{g} / \mathrm{ml}$ to MEPM and $25 \mu \mathrm{g} / \mathrm{ml}$ to IPM. The MICs of PIPC, CAZ, GM and OFLX for the mutant strains were the same as those of parent strain. SDS-PAGE gel analysis of the OMPs of the representative mutant strain showed a marked reduction in a specific OMP with a molecular weight of about 48,000 (data not shown). These results indicated that the mutant resulted from the reduced expression of OprD and they exhibited reduced 
Table 1 Drug susceptibilities of the P. aeruginosa strain and the carbapenem-resistant mutants

\begin{tabular}{|c|c|c|c|c|c|c|c|c|}
\hline \multirow{2}{*}{$\begin{array}{c}\text { Strain } \\
\text { No. }\end{array}$} & \multirow{2}{*}{ Strain ${ }^{a}$} & \multicolumn{7}{|c|}{$\mathrm{MIC}(\mu \mathrm{g} / \mathrm{ml})$} \\
\hline & & DRPM & MEPM & IPM/CS & PIPC & CAZ & GM & OFLX \\
\hline \multirow[t]{2}{*}{ GP 2} & wild type & 0.2 & 0.4 & 1.6 & 100 & 25 & 1.6 & 1.6 \\
\hline & carbapenem-resistant mutant & 3.2 & 6.3 & 12.5 & 100 & 25 & 1.6 & 1.6 \\
\hline \multirow[t]{2}{*}{ GP 3} & wild type & 0.2 & 0.4 & 1.6 & 12.5 & 3.2 & 3.2 & 1.6 \\
\hline & carbapenem-resistant mutant & 3.2 & 6.3 & 25 & 12.5 & 3.2 & 3.2 & 1.6 \\
\hline \multirow[t]{2}{*}{ GP 5} & wild type & 0.8 & 3.2 & 3.2 & 12.5 & 6.3 & 50 & 50 \\
\hline & carbapenem-resistant mutant & 6.3 & 12.5 & 12.5 & 12.5 & 6.3 & 50 & 50 \\
\hline \multirow[t]{2}{*}{ GP10 } & wild type & 0.2 & 0.2 & 1.6 & 3.2 & 3.2 & 1.6 & 1.6 \\
\hline & carbapenem-resistant mutant & 1.6 & 3.2 & 12.5 & 3.2 & 3.2 & 1.6 & 1.6 \\
\hline \multirow[t]{2}{*}{ GP13 } & wild type & 0.1 & 0.1 & 1.6 & 6.3 & 3.2 & 1.6 & 1.6 \\
\hline & carbapenem-resistant mutant & 1.6 & 3.2 & 12.5 & 6.3 & 3.2 & 1.6 & 1.6 \\
\hline \multirow[t]{2}{*}{ GP14 } & wild type & 0.2 & 0.2 & 3.2 & 6.3 & 3.2 & 3.2 & 3.2 \\
\hline & carbapenem-resistant mutant & 1.6 & 3.2 & 25 & 6.3 & 3.2 & 3.2 & 3.2 \\
\hline \multirow[t]{2}{*}{ GP17 } & wild type & 0.1 & 0.1 & 1.6 & 6.3 & 3.2 & 3.2 & 1.6 \\
\hline & carbapenem-resistant mutant & 1.6 & 3.2 & 25 & 6.3 & 3.2 & 3.2 & 1.6 \\
\hline \multirow[t]{2}{*}{ GP33 } & wild type & 0.2 & 0.8 & 0.8 & 25 & 12.5 & 3.2 & 12.5 \\
\hline & carbapenem-resistant mutant & 6.3 & 25 & 12.5 & 25 & 12.5 & 3.2 & 12.5 \\
\hline \multirow[t]{2}{*}{ GP59 } & wild type & 0.1 & 0.4 & 1.6 & 12.5 & 6.3 & 1.6 & 6.3 \\
\hline & carbapenem-resistant mutant & 3.2 & 12.5 & 12.5 & 12.5 & 6.3 & 1.6 & 6.3 \\
\hline \multirow[t]{2}{*}{ GP66 } & wild type & 0.2 & 0.8 & 1.6 & 12.5 & 6.3 & 1.6 & 6.3 \\
\hline & carbapenem-resistant mutant & 3.2 & 12.5 & 12.5 & 12.5 & 6.3 & 1.6 & 6.3 \\
\hline
\end{tabular}

${ }^{a}$ Throughout this study, the drug susceptibilities of the mutant strains isolated in the presence of each selective drug of DRPM, MEPM and IPM were examined. The phenotype of drug susceptibilities of the mutants derived from a given strain were not essentially different in each experiment in this study. The drug susceptibilities of the representative mutant strains are shown in this table.

susceptibility or resistance to DRPM, MEPM, or IPM compared to the parent strain $[3,6,7]$. These results also suggested that both MEPM and IPM could specifically select for the carbapenem reduced susceptible mutants or carbapenem-resistant mutants of $P$. aeruginosa strains, and that DRPM could prevent growth of the mutants at a concentration that would inhibit cell growth. Throughout this study, the term "carbapenem-resistance" was used to where a mutant had reduced susceptibility to carbapenems compared to its parent strain, and not as any indication of absolute MIC.

\section{Plating of Viable Cells of Each Strain on Selective Agar Plates Containing Different Concentrations of Each Carbapenem and the Carbapenem-resistance Level}

To examine the presence of carbapenem-resistant mutants in the bacterial cell population that could arise during cell growth and to show quantitatively the results of Fig. 1, the number of cells of broth culture from each strain that grew

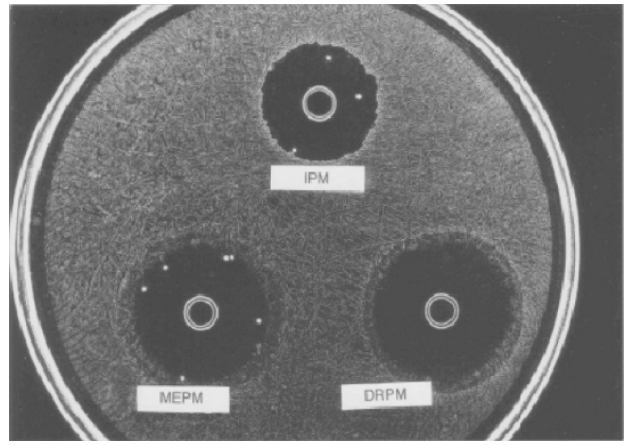

Fig. 1. Presence of carbapenem-resistant mutants within zones of growth inhibition of $P$. aeruginosa around cup containing carbapenem on agar plate.

$0.1 \mathrm{ml}$ of overnight culture of $P$. aeruginosa strain was spread on $A B 3$ agar plate. Three cups were placed on the agar plate. $0.2 \mathrm{ml}$ of $0.8 \mu \mathrm{g} / \mathrm{ml}$ of each carbapenem was put into each cup and the plate was incubated at $37^{\circ} \mathrm{C}$ for 18 hours. Colonies that grew in the clear zone of growth inhibition were purified on a drug free agar plate and resistance levels to carbapenems were examined by agar dilution methods. 
on selective agar plates containing different concentrations of each carbapenem was determined (i.e., population analysis) [15]. The results are shown as a survival curve in Fig. 2.

Almost all inoculated cells from each strain grew on the selective agar plates containing a drug concentration under the strain specific MIC of each carbapenem. The number of bacterial cells of each strain that grew on the selective agar plate containing the strain specific MIC of each carbapenem was around $10^{2}$ to $10^{4}$ cell per ml.

A number of colonies from each strain were grown on selective agar plates containing drug concentrations above the strain specific MIC of each drug. The drug concentration for MEPM ranging from the strain specific MIC to the drug concentration required for the complete inhibition of cell growth in each strain was greater than the drug concentration ranges of DRPM and IPM. The drug concentration range corresponded to the relatively wide zone of incomplete inhibition of cell growth by meropenem between the zone of complete inhibition and the zone of no inhibition of cell growth, as shown in Fig. 1.

Ten colonies of each strain grown on selective agar plates containing half of the concentration of each drug required for the complete inhibition of cell growth in each strain were examined for their drug resistance levels. One or two of the colonies that grew on the DRPM plates of GP2, GP33 and GP59 showed a carbapenem resistance (MIC) that was twice the DRPM concentration for the complete inhibition of cell growth, while the other colonies from each strain had the same level of carbapenem susceptibility as that of the parent strain. The ten colonies grown on the MEPM plates showed carbapenem resistance (MIC) that was the same level as the MEPM concentration for the complete inhibition of cell growth. The ten colonies grown on IPM showed carbapenem resistance (MIC) that was the same or twice the level of the IPM concentration for complete inhibition of cell growth.

The mutants selected with a given carbapenem showed resistance only to carbapenems and were susceptible to other antibiotics (Table 1). The SDS-PAGE gel of OMPs from a representative mutant from each strain showed a marked reduction in the specific OMP of around 48,000 molecular weight (data not shown).

\section{Isolation Frequency of Carbapenem-resistant Mutants of $P$. aeruginosa Strains Grown in Broth without Carbapenem}

The results of the population analysis shown in Fig. 2 implied that the isolation frequencies of carbapenemresistant mutants would be different for the selective drugs DRPM, MEPM, and IPM when mutants are selected at a given concentration of each drug for each strain. The drug concentration for the selective agar plates was half the MIC of DRPM and MEPM, and $1 / 2$ or $1 / 4$ the MIC of IPM for the mutant of each strain.

Carbapenem-resistant mutants of the strains were not selected on selective agar plates containing DRPM, however, they were selected on selective agar plates containing MEPM or IPM at a frequency of around $10^{-7}$ to $10^{-9}$ per cell per generation (Table 2).

The carbapenem-resistant mutants tested from a given parent strain that had been selected with DRPM, MEPM, or IPM had the same resistance phenotype to carbapenems, and susceptibility to other antibiotics as the parent strain (Table 1).

\section{OMPs of P. aeruginosa Strains}

The OMPs of the representative strains and the carbapenem-resistant mutants shown in Table 2 were examined (Fig. 3). The OMPs of the GP3 and GP33 parent strains and the carbapenem-resistant mutants isolated from the selective agar plates containing either DRPM, MEPM, or IPM are shown in Figs. 3A and 3B, respectively. The OMPs of the representative mutant of each strain of the GP2, GP17 and GP66 strains are shown in Figs. 3C, D, and $\mathrm{E}$, respectively. Marked reductions in the concentration of a specific outer membrane protein with a molecular weight of around 48,000 were observed for each of the mutants.

\section{Increased Carbapenem-resistance Level (MIC) of P. aeruginosa Strain after Incubation with Carbapenem} If the carbapenem-resistant mutants arise during growth of the strain in broth culture, and the mutant strain can be increased selectively in the presence of carbapenem, the MIC of the carbapenem for the broth culture will increase beyond that seen for the parent strain.

The maximum resistance levels (MIC) of DRPM were lower than those of MEPM or IPM (the highest for the three carbapenems) for all strains tested (Fig. 4). The drug concentration ranges in broth cultures that gave rise to the maximum-resistance levels to each carbapenem in each strain were different for each of the carbapenems. The maximum resistance level to DRPM for each strain was obtained with broth cultures containing drug concentrations ranging from $1 / 4$ or $1 / 2$ MIC to MIC or twice MIC (Fig. 4). The maximum resistance level to MEPM for each strain was obtained with broth cultures containing drug concentrations ranging from $1 / 4$ or $1 / 2 \mathrm{MIC}$ to greater than MIC, with levels of four, eight or 16 times the MIC for each of the strains being observed (Fig. 4). The maximumresistance level to IPM for each strain was obtained with 

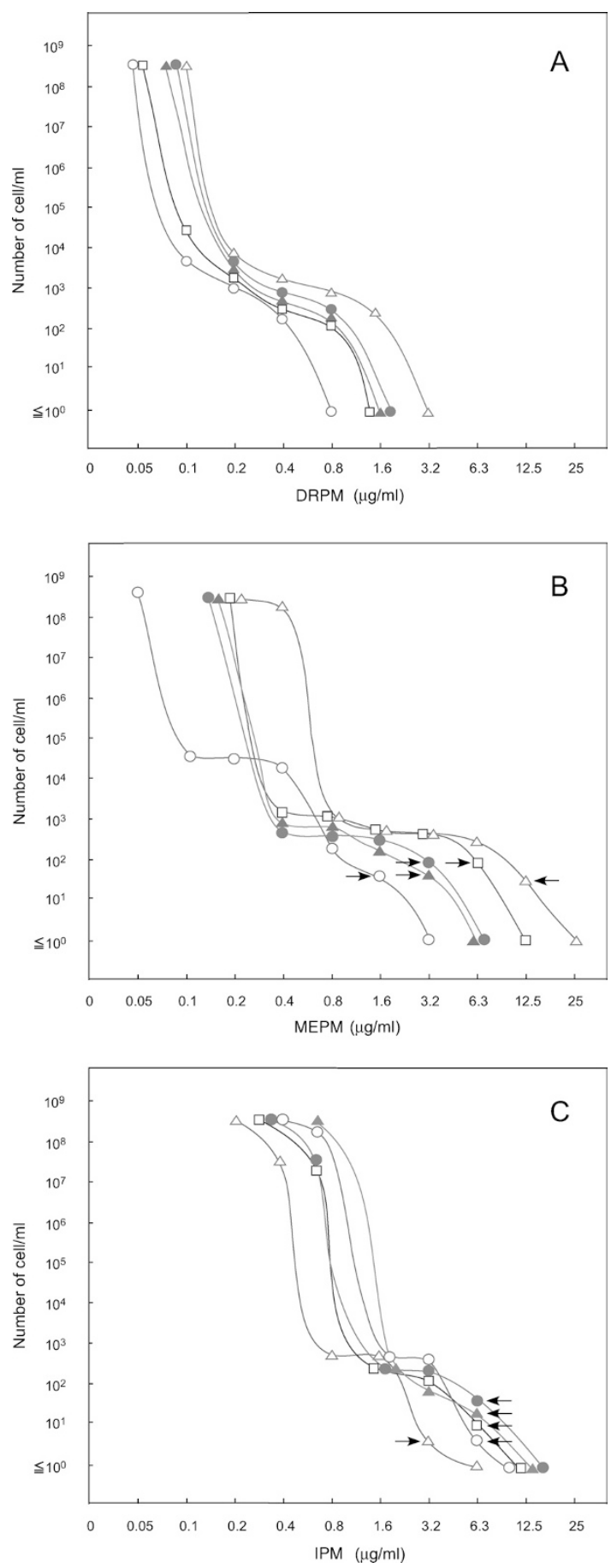

broth cultures containing drug concentrations ranging from 1/2 MIC to MIC, or two to four times the MIC for each of the strains. The drug concentrations of DRPM in the broth culture of each strain needed to select for carbapenemresistant mutants showed a narrower drug concentration range than those of MEPM.

The range of DRPM concentrations required in broth culture to prevent growth of the mutants at the
Fig. 2. Isolation of carbapenem-resistant mutant of $P$. aeruginosa.

Five representative strains are shown. Population analysis of each $P$. aeruginosa strain was carried out. Viable titers and antibiotic resistance levels (population analysis) of bacterial cells from each strain were determined by plating $0.1 \mathrm{ml}$ diluted cultures on an $\mathrm{AB} 3$ agar plate containing different concentrations of each carbapenem. After overnight incubation of the plates at $37^{\circ} \mathrm{C}$, the number of colonies was counted. Ten colonies from each strain that were picked from colonies grown on an agar plate containing one quarter or half of the drug concentration which completely inhibited cell growth of each strain were purified on drug free agar plates three times, and then examined for resistance levels to each carbapenem. The arrow shows that all colonies examined have stable resistance levels to each carbapenem. Viable cell number of each strain grown on selective agar plates containing different concentrations of DRPM (A), MEPM (B), and IPM (C), respectively, were counted. Symbols, O, GP17; $\triangle$, GP33; $\square$, GP59; $\bullet$, GP2; GP3.

concentrations below the DPRM MIC for the carbapenemresistant mutants of each strain were greater than those of MEPM, and equal to or greater than those of IPM.

\section{Discussion}

The mutants of the $P$. aeruginosa strain selected on agar 
Table 2 Isolation frequency of carbapenem-resistant mutants and drug susceptibilities of the mutant strains

\begin{tabular}{|c|c|c|c|c|c|}
\hline \multirow[t]{2}{*}{ Strain No. } & \multirow[t]{2}{*}{ Drug } & \multicolumn{2}{|c|}{$\begin{array}{l}\text { Carbapenem susceptibility of } \\
\text { parent mutant }\end{array}$} & \multirow{2}{*}{$\begin{array}{c}\text { Drug concentration } \\
\text { in selective agar plate }{ }^{a} \\
(\mu \mathrm{g} / \mathrm{ml})\end{array}$} & \multirow{2}{*}{$\begin{array}{l}\text { Isolation frequency of } \\
\text { carbapenem-resistant mutant } \\
\text { (per cell per generation) }\end{array}$} \\
\hline & & $\mathrm{MIC}(\mu \mathrm{g} / \mathrm{ml})$ & $\mathrm{MIC}(\mu \mathrm{g} / \mathrm{ml})$ & & \\
\hline \multirow[t]{3}{*}{ GP2 } & DRPM & 0.2 & 3.2 & 1.6 & $<2 \times 10^{-9}$ \\
\hline & MEPM & 0.4 & 6.3 & 3.2 & $1.74 \times 10^{-8}$ \\
\hline & IPM & 1.6 & 12.5 & 6.3 & $4.81 \times 10^{-8}$ \\
\hline \multirow[t]{3}{*}{ GP3 } & DRPM & 0.2 & 3.2 & 1.6 & $<2 \times 10^{-9}$ \\
\hline & MEPM & 0.4 & 6.3 & 3.2 & $7.95 \times 10^{-7}$ \\
\hline & IPM & 1.6 & 25 & 6.3 & $1.60 \times 10^{-8}$ \\
\hline \multirow[t]{3}{*}{ GP5 } & DRPM & 0.8 & 6.3 & 3.2 & $<2 \times 10^{-9}$ \\
\hline & MEPM & 3.2 & 12.5 & 6.3 & $4.57 \times 10^{-8}$ \\
\hline & IPM & 3.2 & 12.5 & 6.3 & $1.45 \times 10^{-8}$ \\
\hline \multirow[t]{3}{*}{ GP10 } & DRPM & 0.2 & 1.6 & 0.8 & $<2 \times 10^{-9}$ \\
\hline & MEPM & 0.2 & 3.2 & 1.6 & $1.61 \times 10^{-8}$ \\
\hline & IPM & 1.6 & 12.5 & 6.3 & $2.90 \times 10^{-9}$ \\
\hline \multirow[t]{3}{*}{ GP13 } & DRPM & 0.1 & 1.6 & 0.8 & $<2 \times 10^{-9}$ \\
\hline & MEPM & 0.1 & 3.2 & 1.6 & $1.09 \times 10^{-7}$ \\
\hline & IPM & 1.6 & 12.5 & 6.3 & $4.63 \times 10^{-8}$ \\
\hline \multirow[t]{3}{*}{ GP14 } & DRPM & 0.2 & 1.6 & 0.8 & $<2 \times 10^{-9}$ \\
\hline & MEPM & 0.2 & 3.2 & 1.6 & $8.47 \times 10^{-9}$ \\
\hline & IPM & 3.2 & 25 & 6.3 & $2.90 \times 10^{-9}$ \\
\hline \multirow[t]{3}{*}{ GP17 } & DRPM & 0.1 & 1.6 & 0.8 & $<2 \times 10^{-9}$ \\
\hline & MEPM & 0.1 & 3.2 & 1.6 & $8.36 \times 10^{-8}$ \\
\hline & IPM & 1.6 & 25 & 6.3 & $3.07 \times 10^{-8}$ \\
\hline \multirow[t]{3}{*}{ GP33 } & DRPM & 0.2 & 6.3 & 3.2 & $<2 \times 10^{-9}$ \\
\hline & MEPM & 0.8 & 25 & 12.5 & $1.41 \times 10^{-8}$ \\
\hline & IPM & 0.8 & 12.5 & 3.2 & $2.17 \times 10^{-8}$ \\
\hline \multirow[t]{3}{*}{ GP59 } & DRPM & 0.1 & 3.2 & 1.6 & $<2 \times 10^{-9}$ \\
\hline & MEPM & 0.4 & 12.5 & 6.3 & $2.57 \times 10^{-8}$ \\
\hline & IPM & 1.6 & 12.5 & 6.3 & $1.22 \times 10^{-8}$ \\
\hline \multirow[t]{3}{*}{ GP66 } & DRPM & 0.2 & 3.2 & 1.6 & $<2 \times 10^{-9}$ \\
\hline & MEPM & 0.8 & 12.5 & 6.3 & $4.96 \times 10^{-8}$ \\
\hline & IPM & 1.6 & 12.5 & 6.3 & $4.51 \times 10^{-9}$ \\
\hline
\end{tabular}

${ }^{a}$ To determine the drug concentrations for selective agar plates, preliminary experiments were performed several times with different concentrations of each carbapenem for selective agar plates for each strain, and the isolation frequency of the mutant and the carbapenem-resistance levels were examined, and the most competent concentration of each carbapenem for each strain to specifically select the carbapenem-resistant mutant was determined. The data obtained in the experiment shown in Fig. 3 were also used to determine the drug concentration.

plates containing each of the carbapenems exhibited resistance or reduced susceptibility to carbapenems, and showed the same susceptibility to other antibiotics as the parent strain. The mutant strains exhibited a marked reduction in the concentration of a specific outer membrane protein with a molecular weight of about 48,000 . These data indicated that the carbapenems predominantly selected carbapenem-resistant mutants that lacked or had a reduced expression of D2 porin (OprD) [3 7], although there was also a possibility that a mutant with increased expression of MexAB-OprM would be present in the culture [16 19].

The anti-pseudomonas activities of carbapenems are affected by carbapenem-specific porin OprD [3 7] and MexAB-OprM expression [16 22], which have influx and efflux functions, respectively. The loss or reduced expression of OprD of the $P$. aeruginosa strain increases the MICs of the carbapenems [3 7]. Previous studies suggested that the activities of MRPM and DRPM, but 


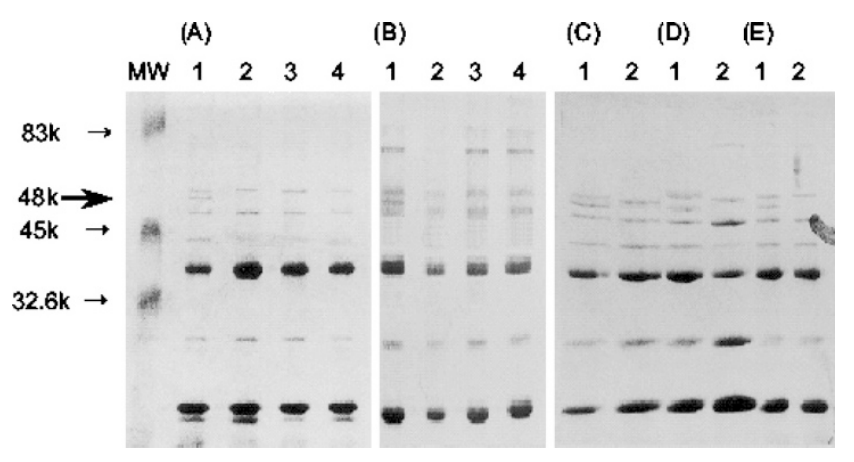

Fig. 3. SDS-PAGE of outer membrane proteins from carbapenem susceptible $P$. aeruginosa strains and the carbapenem-resistant mutants.

(A) P. aeruginosa GP3 and the mutants. lane 1, carbapenem susceptible GP3; lane 2 to lane 4, carbapenem-resistant mutants of GP3 isolated from selective agar plate containing DRPM, MEPM and IPM, respectively. (B) P. aeruginosa GP33 and the mutants. lane 1, carbapenem susceptible GP33; lane 2 to lane 4, carbapenem-resistant mutants of GP33 isolated from selective agar plate containing IPM, MEPM, and DRPM, respectively. (C) lane 1, carbapenem susceptible P. aeruginosa GP2; lane 2, carbapenem-resistant GP2 isolated from agar plate containing DRPM. (D) lane 1, carbapenem susceptible P. aeruginosa GP66; lane 2 carbapenem-resistant GP66 isolated from agar plate containing MEPM. (E) lane 1, carbapenem susceptible $P$. aeruginosa GP17; lane 2 carbapenem-resistant GP17 isolated from agar plate containing IPM; MW, molecular weight marker: bold arrow indicates outer membrane protein with molecular weight around 48,000 . The carbapenem resistant mutant isolated from the selective agar plate containing doripenem was derived from the broth culture containing carbapenem (doripenem) to selectively increase the mutant strain as shown in following experiment described in Fig. 4.

not IPM, are also decreased by the increased production of the efflux protein MexAB-OprM [16, 17, 19, 21], although this mechanism rarely confers outright nor does it confer high level resistance. It confers almost the same level of resistance as is seen in the altered OprD-derivative [19, 21]. This type of mutant is predominantly selected by carbenicillin or CAZ [19]. A multiple resistance mechanism requiring both a lack of OprD for influx and increased expression of MexAB-OprM for efflux would be required to confer a relatively high resistance to MEPM and DRPM for P. aeruginosa [17, 19, 22 24]. This type of mutant has been isolated by selection of a MEPM-resistant mutant from a OprD deficient derivative on agar containing MEPM [23]. This type of double mutant would not be selected readily during carbapenem therapy $[19,21,23$, 24].

The $\mathrm{MIC}_{90}$ values of DRPM for P. aeruginosa strains were one dilution lower than those of MEPM and eight dilutions lower than IPM, indicating that the MICs of IPM were the highest of the three carbapenems $[9,12,19]$. DRPM resembled MEPM, and was more effective than IPM with respect to the MICs for $P$. aeruginosa strains. However, the potency of selection for the mutant cells or the potency of growth prevention of the mutant cells was different for DRPM, MEPM and IPM. A combination of population analysis to determine the presence of the carbapenem-resistant mutant in the culture of each strain (Fig. 1 and Fig. 2), the calculation of the isolation frequency of the carbapenem-resistant mutant on agar containing each carbapenem (Table 2), and experiments to selectively increase the carbapenem-resistant mutant in broth culture containing different concentrations of each carbapenem (Fig. 4) resulted in our drawing the following conclusions: IPM and MEPM selected the carbapenemresistant mutant at the drug concentration that inhibited growth of the parent strain at a frequency of around $10^{-7} \sim 10^{-8}$ per cell of the culture. However, DRPM could not specifically select the mutant strain at a drug concentration capable of inhibiting growth of the parent strain. In contrast to MEPM and IPM, the mutant could not be selected on agar at concentrations of four to 16 times the MIC of DRPM for the parent strain, which were $1 / 2$ MIC for the mutant strains. The maximum drug concentrations of DRPM, MEPM, and IPM in the broth culture that allowed selective increase in carbapenem-resistant mutant in the broth culture were $1 / 8$ to $1 / 16$ MIC of DRPM, $1 / 2$ to $1 / 4$ MIC of MEPM and $1 / 2$ to $1 / 16$ MIC of IPM for the mutant strains, respectively. These data indicated that DRPM has the greatest potency in preventing the emergence of the carbapenem-resistant mutants. The present study suggested that the clinical evaluation of DRPM for the prevention of the emergence of the mutant strain may be worthwhile.

Acknowledgements This work was supported by grants from the Japanese Ministry of Education Culture Sports Science and Technology [Tokuteiryoiki (C), Kiban (B)] and the Japanese Ministry of Health Labor and Welfare (H15-Shinko-9).

\section{References}

1. Watanabe M, Iyobe S, Inoue M, Mitsuhashi S. Transferable imipenem resistance in Pseudomonas aeruginosa. Antimicrob Agents Chemother 35: 147-151 (1991)

2. Senda K, Arakawa Y, Nakashima K, Ito H, Ichiyama S, Shimokata K, Kato N, Ohta M. Multifocal outbreaks of metallo- $\beta$-lactamase-producing Pseudomonas aeruginosa resistant to broad-spectrum $\beta$-lactams, including carbapenems. Antimicrob Agents Chemother 40: 349-353 (1996) 


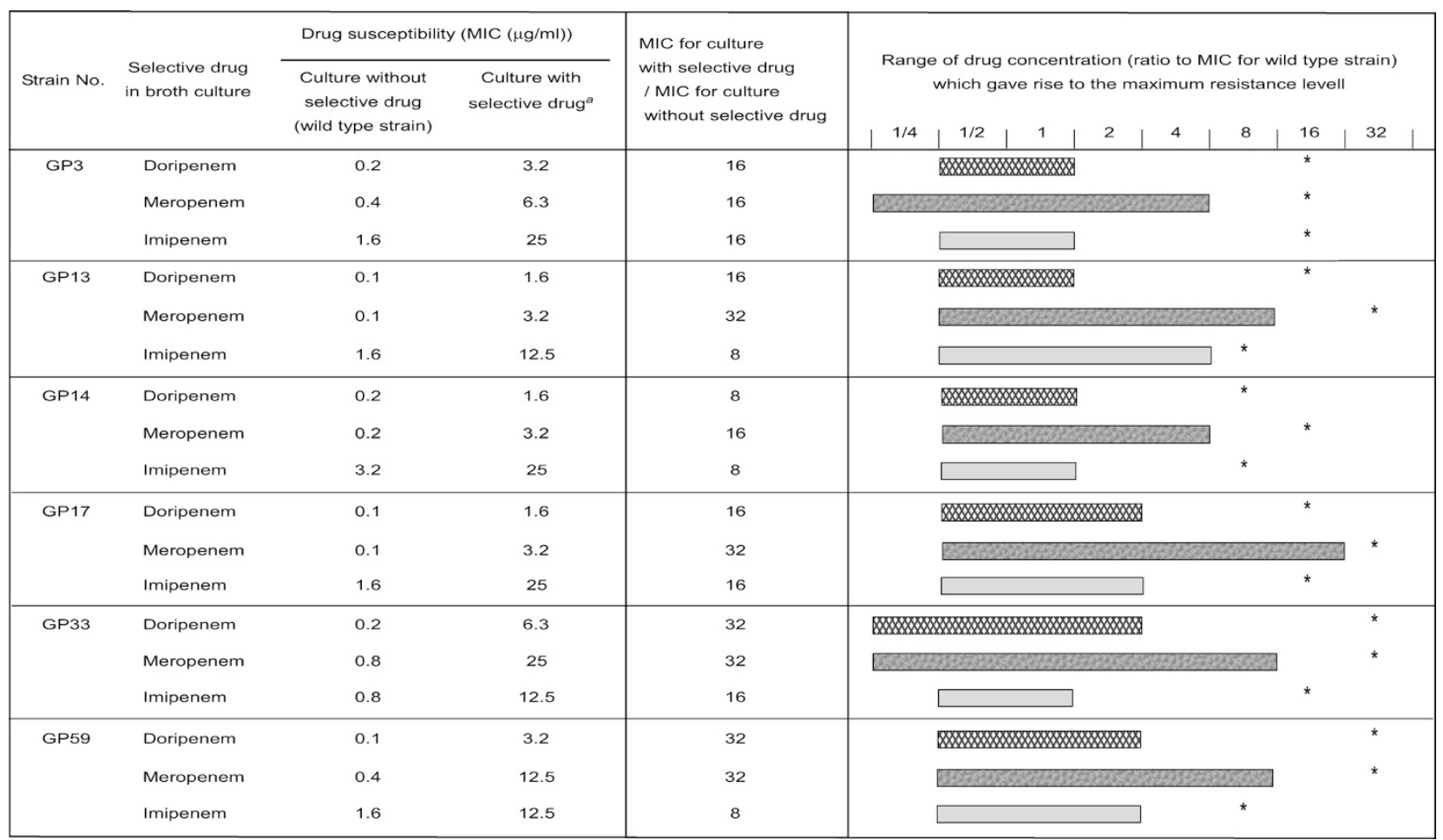

Fig. 4. Increased carbapenem resistance levels of $P$. aeruginosa strains after incubation with carbapenem.

Overnight culture of each strain was diluted appropriately. Approximately $10^{6}$ bacterial cells of each strain were inoculated into $1 \mathrm{ml}$ of AB3 broth containing each different concentrations of each carbapenem and were incubated at $37^{\circ} \mathrm{C}$ for 18 hours with gentle shaking. After incubation, the resistance levels (MICs) to the same carbapenem used in broth culture were examined against each strain using agar dilution methods with around $10^{4}$ inoculated cells. In this experiment, approximately $10^{4} \mathrm{CFU}$ or $10^{6} \mathrm{CFU}$ were inoculated onto each of a series of agar plates containing various antibiotic concentrations. The resistance levels of each strain in the broth culture containing each of the drugs were not essentially different from the different inoculum sizes on the drug plates. The results obtained by inoculating approximately $10^{4}$ cells, are shown in here. ${ }^{a}$ The MIC of each carbapenem in culture with the selective drug indicated the maximum level in the increased MIC for the cultures. Asterisk $\left(^{*}\right)$ in figure indicates drug resistance level (MIC) of each carbapenem for the strain with maximum resistance (i.e., carbapenem-resistant mutant) where the MIC was indicated by ratio of MIC against a culture with selective drug to MIC against the culture without the selective drug (i.e., MIC against the wild type strain).

3. Quinn JP, Dudek EJ, diVincenzo CA, Lucks DA, Lerner SA. Emergence of resistance to imipenem during therapy for Pseudomonas aeruginosa infections. J Infect Dis 154: 289-294 (1986)

4. Büscher KH, Cullmann W, Dick W, Opferkuch W. Imipenem resistance in Pseudomonas aeruginosa resulting from diminished expression of an outer membrane protein. Antimicrob Agents Chemother 31: 703-708 (1987)

5. Lynch MJ, Drusano GL, Mobley HLT. Emergence of resistance to imipenem in Pseudomonas aeruginosa. Antimicrob Agents Chemother 31: 1892-1896 (1987)

6. Trias J, Dufresne J, Levesque RC, Nikaido H. Decreased outer membrane permeability in imipenem-resistant mutants of Pseudomonas aeruginosa. Antimicrob Agents Chemother 33: 1201-1206 (1989)

7. Trias J, Nikaido H. Outer membrane protein D2 catalyzes facilitated diffusion of carbapenems and penems through the outer membrane of Pseudomonas aeruginosa. Antimicrob Agents Chemother 34: 52-57 (1990)
8. Ge Y, Wikler MA, Sahm DF, Blosser-Middleton RS, Karlowsky JA. In vitro antimicrobial activity of doripenem, a new carbapenem. Antimicrob Agents Chemother 48: 1384-1396 (2004)

9. Iso $\mathrm{Y}$, Irie T, Nishino $\mathrm{Y}$, Motokawa K, Nishitani Y. A novel $1 \beta$-methylcarbapenem antibiotic, S-4661. Synthesis and structure-activity relationships of 2-(5-substituted pyrrolidin-3-ylthio)-1 beta-methylcarbapenems. J Antibiot 49: 199-209 (1996)

10. Iso $\mathrm{Y}$, Irie T, Iwaki T, Kii M, Sendo $\mathrm{Y}$, Motokawa $\mathrm{K}$, Nishitani Y. Synthesis and modification of a novel $1 \beta$ methyl carbapenem antibiotic, S-4661. J Antibiot 49: 478-484 (1996)

11. Mikamo H, Izumi K, Hua YX, Hayasaki Y, Sato Y, Tamaya T. In vitro and in vivo antibacterial activities of a new injectable carbapenem, S-4661, against gynaecological pathogens. J Antimicrob Chemother 46: 471-474 (2000)

12. Tsuji M, Ishii Y, Ohno A, Miyazaki S, Yamaguchi K. In vitro and in vivo antibacterial activities of S-4661, a new 
carbapenem. Antimicrob Agents Chemother 42: 94-99 (1998)

13. Stent GS. Molecular biology of bacterial viruses. pp. 188-199. In W. H. Freeman and Co., San Fransisco (1963)

14. Spratt BG. Properties of the penicillin-binding proteins of Escherichia coli K12. Eur J Biochem 72: 341-352 (1977)

15. Sieradzki K, Tomasz A. Inhibition of cell wall turnover and autolysis by vancomycin in a highly vancomycin-resistant mutant of Staphylococcus aureus. J Bacteriol 179: 25572566 (1997)

16. Li XZ, Nikaido H., Poole K. Role of mexA-mexB-oprM in antibiotic efflux in Pseudomonas aeruginosa. Antimicrob Agents Chemother 39: 1948-1953 (1995)

17. Masuda N, Ohya S. Cross-resistance to meropenem, cephems, and quinolones in Pseudomonas aeruginosa. Antimicrob Agents Chemother 36: 1847-1851 (1992)

18. Li XZ, Ma D, Livermore DM, Nikaido H. Role of efflux pump(s) in intrinsic resistance of Pseudomonas aeruginosa: active efflux as a contributing factor to beta-lactam resistance. Antimicrob Agents Chemother 38: 1742-1752 (1994)

19. Mushtaq S, Ge Y, Livermore DM. Doripenem versus Pseudomonas aeruginosa in vitro: activity against characterized isolates, mutants, and transconjugants and resistance selection potential. Antimicrob Agents Chemother 48: 3086-3092 (2004)

20. Masuda N, Sakagawa E, Ohya S, Gotoh N, Tsujimoto H, Nishino T. Substrate specificities of MexAB-OprM, MexCDOprJ, and MexXY-oprM efflux pumps in Pseudomonas aeruginosa. Antimicrob Agents Chemother 44: 3322-3327 (2000)

21. Livermore DM. Of Pseudomonas, porins, pumps and carbapenems. J Antimicrob Chemother 47: 247-250 (2001)

22. Yoneda K, Chikumi H, Murata T, Gotoh N, Yamamoto H, Fujiwara H, Nishino T, Shimizu E. Measurement of Pseudomonas aeruginosa multidrug efflux pumps by quantitative real-time polymerase chain reaction. FEMS Microbiol Lett 243: 125-131 (2005)

23. Köhler T, Michea-Hamzehpour M, Epp SF, Pechere JC. Carbapenem activities against Pseudomonas aeruginosa: respective contributions of OprD and efflux systems. Antimicrob Agents Chemother 43: 424-427 (1999)

24. Carmeli Y, Troillet N, Eliopoulos GM, Samore MH. Emergence of antibiotic-resistant Pseudomonas aeruginosa: comparison of risks associated with different antipseudomonal agents. Antimicrob Agents Chemother 43: 1379-1382 (1999) 